\title{
The independent effects of nausea and vomiting and social support on health-related quality of life during early pregnancy: A longitudinal study
}

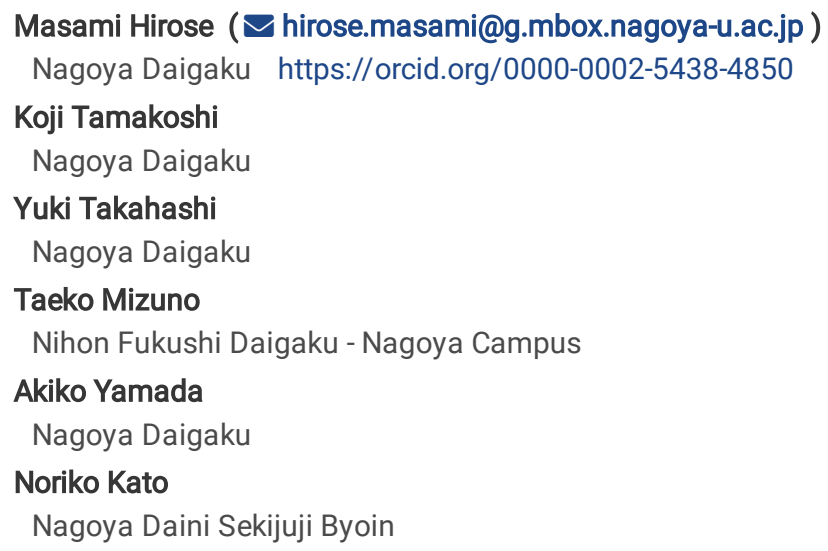

Research article

Keywords: Health-related quality of life, Longitudinal study, Pregnancy, Mixed model, Nausea, Vomiting, Social support

Posted Date: August 31st, 2019

DOI: https://doi.org/10.21203/rs.2.13779/v1

License: (c) (i) This work is licensed under a Creative Commons Attribution 4.0 International License. Read Full License 


\section{Abstract}

Background: Previous studies that have examined the impact of nausea and vomiting (NV) on health-related quality of life (HR-QOL) among early pregnant women have collected data at a single time point, with the timing varying among studies, and even, in one study, among subjects. No previous study has examined the independent association NV and social support have with HR-QOL. We conducted a longitudinal study from August 2018 to February 2019 to investigate the independent association NV and social support have with HR-QOL among early pregnant women using repeated-measurement data. Subjects were 153 pregnant women who were 20 years of age or older and at less than 20 weeks of gestation at their first prenatal visit. They were recruited from a perinatal outpatient unit of a general hospital. Methods: Along with providing sociodemographic data, subjects were asked to complete three self-administered questionnaires: the Index of Nausea, Vomiting, and Retching (INVR); the Multidimensional Scale of Perceived Social Support (MSPSS); and the 12-item Short Form Health Survey (SF-12). Further, at every subsequent visit up to 20 weeks of gestation, they were asked to complete both the SF-12 and the INVR (giving a maximum of three survey completions). Results: After controlling for internal correlations and confounding factors using a mixed model for longitudinal data, the INVR score was found to be significantly negatively associated with the physical component summary scale score of the SF-12; however, the MSPSS score showed no association with the physical component summary scale score. On the other hand, both the INVR score and the MSPSS score were negatively and positively, respectively, significantly associated with the mental component summary scale score of the SF-12, independently of each other. Conclusions: The severity of NV significantly impacts physical quality of life during early pregnancy, but social support has no such impact. However, NV and social support independently and significantly affect mental quality of life, independently of each other. Health professionals should recognize the significant impact of NV on HR-QOL. Additionally, they should be aware that social support contributes to the improvement of mental quality of life, regardless of the degree of NV.

\section{Background}

Nausea and vomiting in pregnancy (NVP) is a common, normal condition, with $50-80 \%$ of pregnant women experiencing its symptoms during their first trimester [1]. Clinically, NVP is often regarded as evidence that pregnancy is progressing normally and is not considered an illness unless the symptoms are severe [2]. However, NVP may affect physiological, psychological, and social aspects of pregnant women's lives and may reduce their quality of life $(\mathrm{QOL})$. In recent years, the association between QOL and pregnancy-related symptoms, including NVP, has been receiving increasing attention. Previous studies have reported that pregnant women who experience NVP during their first trimester have significantly lower physical and mental component summary scores, as measured using the 12- or 36-item Short Form Health Survey; SF-12 and SF-36, respectively $[3,4,5]$. Meanwhile, other studies have found NVP to be negatively associated only with the physical domain of QOL $[6,7]$. The inconsistency among these studies may be due to differences in study design, particularly the timing of data collection. To our knowledge, all these previous studies only collected data on NVP, QOL, and other factors from the subjects at one time point during the first trimester; additionally, the timing of this collection varied among studies and in one study even differed among subjects. In general, symptoms of NVP appear at 4-6 weeks of gestation, peak at 8-12 weeks, and spontaneously disappear by 20 weeks [8, 9]. Therefore, in order to accurately understand the relationship between NVP and QOL, it is necessary to observe subjects longitudinally and obtain relevant data on multiple occasions.

Social support is also considered to be an important factor affecting QOL in early pregnancy. As far as we can determine, only two studies have examined this relationship. Elsenbruch et al. [10] (2006) demonstrated, through a bivariate analysis of 896 women with an average gestational age of 8.2 weeks, that low social support is statistically significantly associated with reduced QOL. Meanwhile, using multivariate regression analysis, Nicholson et al. [11] examined 175 women with an average gestational age of 14.6 weeks and reported that the presence of social support from a significant other was significantly associated with increased QOL. However, neither of these two studies considered the impact of NVP on QOL.

Thus, to address the existing gap in the literature and to find more robust evidence that can help perinatal healthcare professionals develop and adopt appropriate strategies, in the present paper we conducted a follow-up study during early pregnancy.

\section{Methods}

\section{Aims}

The aims of this study were to clarify the patterns of NVP and QOL during early pregnancy and to elucidate the independent association that NVP and social support have with QOL among early pregnant women.

\section{Study design, setting, and subjects}

A prospective longitudinal study was conducted between August 2018 and February 2019. A sample of pregnant women was recruited from women who visited the maternity unit of a general hospital in Nagoya City, Japan, for their first pregnancy checkup. The following inclusion criteria were applied: 1) singleton pregnant women aged 20 years or older; 2) the ability to read and write in Japanese; and 3) permitted by an obstetrician to participate in this study, based on the results of the initial pregnancy checkup. 


\section{Procedure}

During face-to-face interviews in the maternity unit, a researcher informed the subjects of the purpose of this study and their right to refuse to participate in and to withdraw from the study at any time. After obtaining subjects' written informed consent, we asked them to complete an initial survey instrument that included several questions regarding their sociodemographic status, the 12-item Short Form Health Survey (SF-12), the Index of Nausea, Vomiting, and Retching (INVR), and the Multidimensional Scale of Perceived Social Support (MSPSS). At every subsequent visit until they had reached 20 weeks of gestation, the subjects were asked to complete both the SF-12 and INVR again (giving a maximum of three completions of the scales). If a subject encountered pregnancy complications or was admitted to hospital, she was excluded from the study.

As the gestational age at the first and subsequent visits differed among subjects, we divided the gestational ages at which the subjects answered the questionnaire into three periods (5-8 weeks of gestation: G1; $9-12$ weeks: G2; $13-20$ weeks: G3); this stratification was based on the findings of previous studies regarding the fluctuation of NVP $[8,9]$.

The study protocol was approved by the ethics committee of the Nagoya University School of Medicine (Approval Number 17-157) and Japanese Red Cross Nagoya Daini Hospital.

\section{Measurements}

\section{Health-related quality of life (HRQOL)}

Although several specialized QOL instruments have been developed, the Short Form-36 Health Survey (SF-36) is widely used as a standard measure for evaluating QOL among early pregnant women [1]. Specifically, the SF-36 is a generic measurement tool for HRQOL that has proven validity and reliability [12]. The 12-item Short Form Health Survey (SF-12) is a shortened version of the SF-36. Both SF-12 and SF-36 have previously been translated into Japanese and have been used for international comparisons of HRQOL assessments regarding patients with various diseases [13]. SF-12 comprises a physical component summary scale (PCS) and a mental component summary scale (MCS), which are used to assess physical and mental well-being, respectively [14]. Scores for these summary measures range from 0 to 100 . The SF-12 is highly reliable and has shown very good criterion validity when compared to the gold standard of HRQOL, the SF-36 [15]. In this study, we used an acute version of the SF-12 with a one-week recall period in order to identify short-term changes in HRQOL. For this study, Cronbach's alpha coefficients were $0.879,0.897$, and 0.901 at G1, G2, and G3, respectively.

\section{Index of Nausea, Vomiting, and Retching}

The Index of Nausea, Vomiting, and RetchingINVR) was originally developed as a scale for measuring the degree of nausea and vomiting among cancer patients receiving chemotherapy $[16,17]$. However, subsequent researchers have modified it into a reliable and valid scale for measuring NVP [18]. The INVR contains exactly the same questions as does the conventional Rhodes Index of Nausea, Vomiting Form 2 (INV-2), with the only modifications being to the phrasing of questions in order to make them easier to answer. The eight-item INVR measures the self-reported severity of nausea, vomiting, and retching in the last 12 hours, with each item rated on a five-point Likert scale. INVR scores range from 0 to 32 , and the higher the total score, the greater the severity of nausea, vomiting, and retching. The score ranges of $1-8,9-16$, and 17-32 are classified as mild, moderate, and severe NVP, respectively. The Japanese version of INV-2 has been proven to be valid and reliable [19], with its validity and reliability being particularly high among pregnant women with NVP [7]. Iwakuni [20] (2017) created the Japanese version of INVR using the same process by which the original INVR was created, and Cronbach's alpha coefficient for the original Japanese version of INVR was 0.82 . For the present study, Cronbach's alpha coefficient was 0.87 .

\section{Multidimensional Scale of Perceived Social Support}

The Multidimensional Scale of Perceived Social Support (MSPSS) was developed to assess subjective social support [21]. The MSPSS is a 12-item self-report questionnaire; its items are scored on a seven-point Likert scale ranging from 1 to 7 , meaning total scores range from 12 to 84 . The MSPSS comprises three subfields: perception of support from family, friends, and significant other, respectively; higher scores indicate greater perceived social support. The MSPSS has been translated into Japanese [22], and consequent Cronbach's alpha coefficients were 0.94, 0.90, 0.88, and 0.92 for the support from family, friends, and significant other subscales, and the scale in total, respectively. In this study, the Cronbach's alpha coefficients were $0.89,0.92,0.85$, and 0.92 for the respective subscales.

\section{Sociodemographic data collection}


Sociodemographic data were collected to examine their potential influence on the study variables. Data collected included age, marital status (unmarried or married), parity (primipara or multipara), education level (high school and below or college and above), occupation (housewife or employed outside the home), and household income (less than five million yen or more than five million yen).

\section{Statistical analyses}

First, patterns of NVP (INVR score) and QOL (PCS and MCS scores derived from the SF-12) were analyzed. Without considering longitudinal data, crude mean scores and standard deviations of INVR, PCS, and MCS were calculated for the three periods, and differences in INVR, PCS, and MCS scores across the three periods were compared using a one-way ANOVA with Bonferroni correction. Furthermore, estimated mean scores and standard errors were calculated using a linear mixed model with consideration of continuous longitudinal data, which facilitated full use of the available data while controlling for internal correlations and potential confounding variables. Second, Pearson's product- moment correlation coefficients were used to evaluate the strength of the monotonic association between the QOL score (PCS and MCS) and INVR and MSPSS scores. Finally, to estimate the independent contributions of NVP and social support to QOL during early pregnancy, we calculated fixed-effects parameter estimates and their 95\% confidence intervals using a linear mixed model, with the QOL score (PCS and MCS derived from SF-12) set as a dependent variable. To remove confounding effects, four different models were created for this study. Model I had indicator variables for $\mathrm{G} 2$ and G3; G1 was set as a reference and sociodemographic variables, including age (continuous), marital status, parity, education level, occupation, and household income, were used to examine the pattern of the QOL score, after adjusting for sociodemographic variables. Model II included the INVR score, in addition to the variables used in Model I, and was designed to examine NVP's association with QOL, after adjusting for sociodemographic variables. Model III contained the MSPSS score, as well as the variables used in Model I, and was designed to examine the association between social support and QOL, after adjusting for sociodemographic variables. Finally, Model IV included both the INVR score and the MSPSS score, along with the variables used in Model I. The above-mentioned four models were performed by adopting the PCS score and the MCS score separately as dependent variables.

All analyses were performed using IBM SPSS Statistics 25.0 for Windows (IBM Corp., Armonk, NY), with p < 0.05 considered to indicate statistical significance.

\section{Results}

Of the 175 pregnant women recruited, 22 were excluded from the study; one was excluded because of depression, four because of hospitalization for hyperemesis gravidarum, four because of hospitalization for threatened abortion, six because of spontaneous abortion, and seven because of incomplete questionnaires. Finally, the data of a total of 153 pregnant women were analyzed in this study.

Table 1 presents the sociodemographic characteristics of the remaining 153 subjects. The mean age of the study subjects was $33.9 \pm 4.8$ years. Overall, 146 (95.4\%) were married, 78 (51.0\%) were primipara, 87 (56.9\%) had an education level of college or above, 107 (69.9\%) were employed, and 107 (69.9\%) had a household income of five million yen or more.

After the subjects had been divided into groups based on the gestational ages at which they answered the questionnaire, we found that 11 subjects had provided data for all three periods. Meanwhile, the numbers of subjects who provided data for G1 and G2, G1 and G3, and G2 and G3 were 27, 1 , and 94 , respectively. The numbers of those who provided data at only one point were one, 17 , and two for G1, G2, and G3, respectively.

Table 2 shows how INVR, PCS, and MCS scores fluctuate with gestational age during the early pregnancy period. Regarding the degree of NVP, as indicated by the INVR score, the proportion of women with severe NVP was highest at G2 (5.0\%, 10.1\%, and 4.7\% at G1, G2, and G3, respectively), and the number of subjects with no NVP increased with gestational age (5.0\%, $16.8 \%$, and $41.1 \%$ at G1, G2, and G3, respectively). Further, the crude value of INVR decreased sharply from G2 to G3; however, both PCS and MCS values increased from G2 to G3. These tendencies did not change after adjustment for confounding factors using a mixed model.

Table 3 demonstrates the Pearson's correlation coefficients among the INVR, MSPSS, PCS, and NCS scores in terms of the three periods of G1, G2, and G3. Here, the INVR score was found to be significantly and negatively associated with both PCS and MCS scores during G2 and G3, but not during G1 (PCS: $r=-0.305, p=0.055$; MCS: $r=-0.260, p=0.105$ ). Meanwhile, the association between the MSPSS score and the PCS score was not statistically significant, but a significant and positive association between MSPSS score and MCS score was observed at G1, G2, and G3. There was no significant association between the INVR score and MSPSS score.

Table 4 shows the relationships INVR and MSPSS had with PCS, which were determined using mixed models for longitudinal data. Here, the PCS score was significantly higher at G3 (13-20 weeks of gestation) compared with G1 (5-8 weeks of gestation), after adjusting for confounding factors including age, marital status, parity, education level, occupation, and household income (Model I). When added as an independent variable to Model I (Model II), INVR was found to be significantly and negatively associated with the PCS score. Similarly, MSPSS was also added to Model I (Model III), but did not show any significant association with PCS. Finally, when both INVR and MSPSS were simultaneously added to Model I (Model IV), only INVR showed a statistically significant and negative association with PCS.

Page $4 / 10$ 
The relationships INVR and MSPSS had with MCS are presented in Table 5. Here, as with PCS, the MCS score was significantly higher at G3 compared with G1, after adjusting for confounding factors. When added as an independent variable (Model II), INVR was found to be significantly and negatively associated with the MCS score. Further, when only MSPSS was added to the model (Model III), MSPSS showed a significant and positive association with MCS. Finally, when INVR and MSPSS were simultaneously added to Model I (Model IV), both showed a significant and independent association with MCS (regression coefficient, lower 95\% confidence limit, and upper $95 \%$ confidence limit for INVR: -0.49 , -0.66 , and -0.31 , respectively; values for MSPSS: $0.30,0.19$, and 0.42 , respectively).

The above-mentioned analyses were then performed on the data that were available for at least two periods, and the same findings were obtained.

\section{Discussion}

This is the first study to examine the effects that NVP and social support have on HRQOL during early pregnancy using longitudinal data. Using validated measures for collecting data and mixed models for analyzing the data, which made all data available while controlling for internal correlations and other confounding variables, our longitudinal within-subject study with multiple follow-up time points provided clear findings. When early pregnancy was divided into three periods, G1 (5-8 weeks of gestation), G2 (9-12 weeks of gestation), and G3 (13-20 weeks of gestation), NVP tended to ease at G3, while HRQOL tended to improve at G3. After adjusting for some confounding factors, including age, marital status, parity, education level, occupation, and household income, NVP was significantly and negatively associated with physical QOL; however, social support showed no significant association. On the other hand, both NVP and social support, considered independently of each other, were determined to be significantly associated with mental QOL.

Regarding the fluctuation of NVP during early pregnancy, several studies have reported that NVP typically appears at approximately five weeks' gestation, peaks during 8-12 weeks' gestation, and spontaneously disappears by 20 weeks' gestation [8, 9]. In our study, after adjusting for sociodemographic factors, we found no significant difference in the INVR score between G1 and G2 but found the INVR score at G3 to be significantly lower than those at G1 and G2. Categorizing the subjects into four groups ranging from no NVP to severe NVP, the respective proportions were 2/40 (5.0\%; no NVP) and 2/40 (5.0\%; severe NVP) at G1, and 25/149 (16.8\%; no NVP) and 15/149 (10.1\%; severe NVP) at G3. During G2, some women can feel better while others' NVP can become severe. In the future, it will be necessary to examine the factors that cause this difference. Regarding the change in HRQOL, both physical and mental QOL were significantly higher at G3 than at G1 and G2. Further, significant and negative associations between INVR and the PCS and MCS scores were observed at G1, G2, and G3. This indicates that NVP has a strong impact on QOL during early pregnancy.

Few studies have investigated the relationship between NVP and QOL during the first trimester. Previously, Lacasse et al. [3] examined 367 pregnant women whose gestational ages were up to 16 weeks, Chan et al. [4] examined 396 pregnant women who were between 10 and 14 weeks of gestation, and Bai et al. [5] examined 5,079 pregnant women who were at 18 weeks of gestation. All three studies reported that NVP has a significant negative association with both physical and mental QOL. On the other hand, Tan et al. [6], who examined 116 pregnant women who were at 9-16 weeks of gestation, and Kugahara and Ohashi [7], who examined 162 pregnant women and divided the gestation period into four phases, reported that NVP is significantly and negatively associated with physical QOL alone, and not significantly associated with mental QOL. The reason these research results are not consistent may be that data were obtained from the subjects at only one time point, during the early pregnancy period; furthermore, the number of weeks of data collection differed among studies and even within one study. In contrast, in our study, using longitudinal data, we found NVP to affect both physical QOL and mental QOL in early pregnancy.

Some previous studies have examined the association between social support and HRQOL among pregnant women, but few have targeted women in early pregnancy. Elsenbruch et al. [10] examined pregnant women at four to 12 weeks of gestation and reported that social support is positively associated with both PCS and MCS in SF-12. However, this study did not consider sociodemographic factors. On the other hand, Nicholson et al. [11], who examined 175 pregnant women with an average gestational age of 14.6 weeks, reported that, after consideration of confounding factors, support from significant others is positively associated with the mental health subscale of SF-36, but not with the tool's physical functioning subscale. However, neither of these two studies considered NVP, which strongly affects HRQOL in early pregnancy.

In our study, social support showed no significant association with physical QOL. This finding may be explained by the absence of an association between MSPSS and INVR throughout G1 to G3. The most interesting finding from this study is that social support is positively associated with mental QOL, independently of NVP. Poor mental health in early pregnancy is known to be associated with depressive tendencies, which may affect the mental status of pregnant women and their attachment to the fetus during the subsequent pregnancy period [11, 23, 24]. Our findings suggest that social support can provide mental stability for early pregnant women, even if NVP is severe.

\section{Strengths and limitations}

The strengths of the present study include its longitudinal design. Using a mixed model, we analyzed data obtained at multiple time points during early pregnancy. Moreover, since data on many kinds of factors known or suspected to influence HRQOL were collected, through multivariate 
adjustment we could elucidate the independent effects of NVP and social support on HRQOL. In addition, regarding the main outcome and exploratory variables, we were able to evaluate HRQOL, NVP, and social support using validated measurements and, thus, avoid information bias. Nevertheless, several limitations to this study deserve mention. The subjects were pregnant women who attended a health checkup at one of the core hospitals in a large city in Japan. Consequently, our findings may not be generalizable. Second, our study was designed to longitudinally obtain multiple data from subjects, but there were subjects with missing values because the health-check intervals differed among subjects. To address this problem, we adopted a mixed model that allowed us to produce unbiased results when missing data were present. Third, information regarding social support was obtained only at the first visit. Depending on the condition of the pregnant woman, social support situations may change during the early months of pregnancy. However, it is worth noting that using the Brief Social Support Questionnaire, Chou et al. [25] reported no change in social support over the three trimesters.

\section{Conclusion}

Using longitudinal data for pregnant women at 5-20 weeks of gestation, we found robust evidence regarding the influence NVP and social support have on HRQOL. Comparing groups based on gestational age, we found that NVP tended to ease after 13 weeks of gestation. When the 5-8 weeks of gestation group and the 9-12 weeks of gestation group were compared, we found that some subjects had improved their NVP over 9-12 weeks, while others' NVP had become severe. Both physical and mental QOL tended to increase after 13 weeks of gestation. The severity of NVP had a significant impact on physical QOL, but social support showed no such impact. On the other hand, NVP and social support significantly affected mental QOL independently of each other. Health professionals should recognize the significant impact of NVP on HRQOL and implement optimal management of NVP. Additionally, they should be aware that social support contributes to the improvement of mental QOL, regardless of the degree of nausea and vomiting, and should try to support pregnant women themselves. They also need to convey to people around pregnant women (e.g., family and friends) the importance of providing support. Moreover, in future, the efficacy of care and treatment for NVP and levels of social support should be evaluated from the viewpoint of identifying means of recovering pregnant women's QOL.

\section{List Of Abbreviations}

Cl: Confidence Interval; Health-Related Quality of Life (HRQOL); INVR: Index of Nausea, Vomiting, and Retching; MCS: Mental Component Summary scale; MSPSS: Multidimensional Scale of Perceived Social Support; NV: Nausea and vomiting; Nausea and vomiting in pregnancy (NVP); PCS: Physical Component Summary scale;SF-12: 12-item Short Form Health Survey

\section{Declarations}

\section{Authors' contributions}

$\mathrm{MH}$ was responsible for the study design, data collection, data analysis, and redaction of the manuscript. KT was responsible for the study design, provided statistical advice, and redacted the manuscript. NK was responsible for this study design, data collection. YT, TM, and AY conceived of the idea for this paper. All authors read and approved the final manuscript.

\section{Ethics approval and consent to participate}

The study protocol was approved by the ethics committee of the Nagoya University School of Medicine (Approval Number 17-157).

\section{Consent for publication}

Not applicable.

\section{Availability of data and material}

The datasets used and analyzed during the current study are available from the corresponding author on reasonable request.

\section{Competing interests}

The authors declare that they have no competing interests.

\section{Funding}


Not applicable.

\section{Acknowledgements}

We would like to thank all study subjects and the staff of the Department of Obstetrics and Gynecology, Japanese Red Cross Nagoya Daini Hospital, Japan.We would also like to thank Editage (www.editage.com) for English language editing.

\section{References}

1. Matthews A, Haas DM, O’Mathúna DP, Dowswell T. Interventions for nausea and vomiting in early pregnancy. Cochrane Database Syst Rev. 2015;9:CD007575.

2. Hinkle SN, Mumford SL, Grantz KL, Silver RM, Mitchell EM, Sjaarda LA, et al. Association of Nausea and Vomiting During Pregnancy With Pregnancy Loss. JAMA. 2016;176(11):1621-7.

3. Lacasse A, Rey E, Ferreira E, Morin C, Bérard A. Nausea and vomiting of pregnancy: What about quality of life? BJOG. 2008;115(12):14841493.

4. Chan OK, Sahota DS, Leunge TY, Chan LW, Fung TY, Lau TK. Nausea and vomiting in health-related quality of life among Chinese pregnant women. Aust. N. Zeal. J. Obstet. Gynecol. 2010;50(6):512-8.

5. Bai G, Korfage IJ, Groen EH, Jaddoe VW, Mautner EM, Raat H. Associations between nausea, vomiting, fatigue and health-related quality of life of women in early pregnancy: The Generation R Study. PLOS ONE. 2016;11(11):e0166133.

6. Tan A, Lowe S, Henry A. Nausea and vomiting of pregnancy: Effects on quality of life and day-to-day function. Aust N Z J Obstet Gynecol. 2017;58(3):278-290.

7. Kugahara T, Ohashi K. Characteristics of nausea and vomiting in pregnant Japanese women. Nurs Sci. 2006;8(3):179-184.

8. Sherman PW, Flaxman SM. Nausea and vomiting of pregnancy in an evolutionary perspective. The Am. J. of Obstet. Gynecol. 2002;186:S1907.

9. Deucher N. Nausea and vomiting in pregnancy: A review of the problem with particular regard to psychological and social aspects. BJOG. 1995;102(1):6-8.

10. Elsenbruch S, Benson S, Rücke M, Rose M, Dudenhausen J, Pincus-Knackstedt M, Arck P. 2006. Social support during pregnancy: Effects on maternal depressive symptoms, smoking and pregnancy outcome. Hum. Reprod. 2006;22(3):869-877.

11. Nicholson WK, Setse R, Hill-Briggs F, Cooper LA, Strobino D, Powe NR. Depressive symptoms and health-related quality of life in early pregnancy. Obstet Gynecol. 2006;107(4):798-806.

12. Ware EJ. Standards for validating health measures: Definition and content. J. Chronic Dis. 1987;40(6):473-480.

13. Fukuhara S, Bito S, Green J, Hsiao A, Kurokawa K. Translation, adaptation, and validation of the SF-36 Health Survey for use in Japan. J Clin. Epidemiol. 1998;51(11):1037-1044.

14. Gandek B, Ware JE, Aaronson NK, Apolone G, Bjorner JB, Brazier JE, Kaasa S, Leplege A, Prieto L, Sullivan M. Cross-validation of item selection and scoring for the SF-12 Health Survey in nine countries. J. Clin. Epidemiol. 1998;51(11):1171-8.

15. Ware Jr JE, Kosinski M, Keller SD. A 12-item short-form health survey: Construction of scales and preliminary tests of reliability and validity. Medical Care. 1996;34(3):220-233.

16. Rhodes VA, Watson PM, Johnson MH. Development of reliable and valid measures of nausea and vomiting. Cancer Nurs. 1984;7(1), 33-42.

17. Rhodes VA, McDaniel RW. The Index of Nausea, Vomiting, and Retching: A new format of the Index of Nausea and Vomiting. Oncol Nurs Forum. 1999;26(5):889-894.

18. Zhou Q, O'Brien B, Soeken K. Rhodes Index of Nausea and Vomiting-Form 2 in Pregnant Women. A confirmatory factor analysis. Nurs. Res. 2001;50(4):251-7.

19. Arakawa S. Relaxation to reduce nausea, vomiting, and anxiety induced by chemotherapy in Japanese patients. Cancer Nurs. 1997;20(5):3429.

20. Iwakuni A. Evaluation of the effects a nursing care program for improving self-care behavior through encouraging patients to find their own ways of alleviating nausea and vomiting in pregnancy has on self-care agency and nausea and vomiting among pregnant women. Jpn J. Nurs Sci. 2017;37:353-363.

21. Zimet GD, Dahlem NW, Zimet SG, Farley GK. The multidimensional scale of perceived social support. J. Pers. Assess. 1988;52(1):30-41.

22. Iwasa H, Gondou Y, Masui Y, Inagaki H, Kawaai C, Ohtsuka R, et al. Reliability and validity of the Japanese version of the Social Support Scale. J. Health Welfare Stat. 2007;54(6):26-33 (in Japanese).

23. Nieto L, Lara MA, Navarrete L. Prenatal predictors of maternal attachment and their association with postpartum depressive symptoms in Mexican women at risk of depression. Matern. Child Health J. 2017;21(6):1250-9.

Page $7 / 10$ 
24. McCormack D, Scott-Heyes G, McCusker CG. The impact of hyperemesis gravidarum on maternal mental health and maternal-fetal attachment. J Psychosom Obstet Gynaecol. 2011;32(2):79-87.

25. Chou F, Kuo S, Wang R. A longitudinal study of nausea and vomiting, fatigue and perceived stress in, and social support for, pregnant women through the three trimesters. Kaohsiung J Med Sci. 2008;24(6):306-314.

\section{Tables}

Table 1. Subjects' sociodemographic characteristics $(n=153)$

\begin{tabular}{|c|c|c|}
\hline & Mean/n & $\mathrm{SD} / \%$ \\
\hline Age (years) & 33.9 & 4.8 \\
\hline \multicolumn{3}{|l|}{ Marital status } \\
\hline Married & 146 & 95.4 \\
\hline Unmarried & 7 & 4.6 \\
\hline \multicolumn{3}{|l|}{ Parity } \\
\hline Primipara & 78 & 51.0 \\
\hline Multipara & 75 & 49.0 \\
\hline \multicolumn{3}{|l|}{ Highest education level } \\
\hline High school and below & 66 & 43.1 \\
\hline College and above & 87 & 56.9 \\
\hline \multicolumn{3}{|l|}{ Employed } \\
\hline No & 46 & 30.1 \\
\hline Yes & 107 & 69.9 \\
\hline \multicolumn{3}{|l|}{ Household income } \\
\hline$<5$ million yen & 33 & 21.6 \\
\hline$\geq 5$ million yen & 107 & 69.9 \\
\hline No answer & 13 & 8.5 \\
\hline
\end{tabular}

Data are means \pm standard deviation (SD) or numbers (\%)

Table 2. Fluctuations of INVR, PCS, and MCS scores with gestational age during early pregnancy

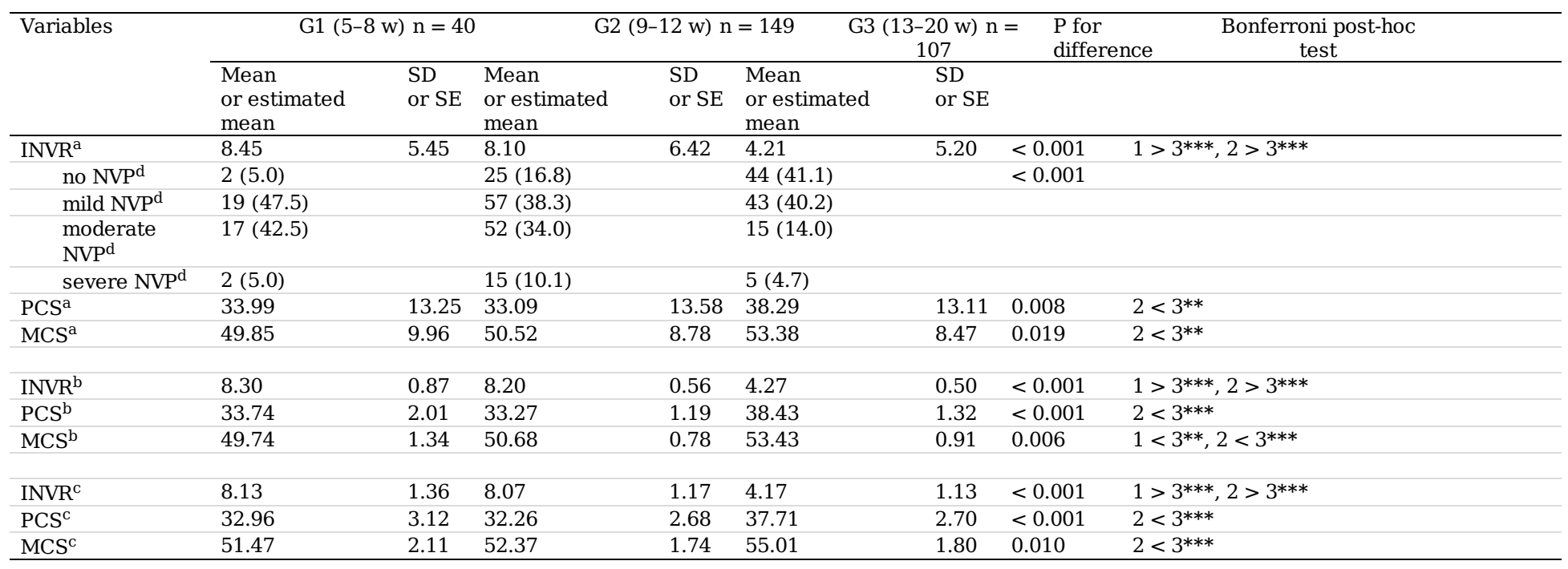

INVR: Index of Nausea, Vomiting, and Retching; PCS: Physical Component Summary scale; MCS: Mental Component Summary scale.

a Crude mean and standard deviation. The difference among the three categories of gestational age (G1: 5-8 weeks, G2: 9-12 weeks, and G3: 13-20 weeks) was evaluated using a one-way analysis of variance.

b Estimated mean and standard error, calculated using a linear mixed model, and with the categories of gestational age set as the independent variable. 


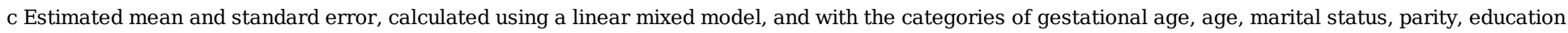
level, occupation, and household income as the independent variables.

d Number (\%). The difference among the three categories was evaluated using a chi-square test.

$* * \mathrm{p}<0.01, * * * \mathrm{p}<0.001$

Table 3. Pearson's correlation coefficients among INVR, MSPSS, PCS, and MCS scores (G1, G2, G3)

\begin{tabular}{|c|c|c|c|c|c|c|}
\hline \multirow[t]{2}{*}{ Variables } & \multicolumn{2}{|c|}{ G1 (5-8 w) } & \multicolumn{2}{|c|}{ G2 ( 9-12 w) } & \multicolumn{2}{|c|}{ G3 $(13-20 \mathrm{w})$} \\
\hline & $\mathrm{r}$ & $\mathrm{p}$ & $\mathrm{r}$ & $\mathrm{p}$ & $\mathrm{r}$ & $\mathrm{p}$ \\
\hline INVR and PCS & -0.305 & 0.055 & -0.434 & $<0.001$ & -0.389 & $<0.001$ \\
\hline INVR and MCS & -0.260 & 0.105 & -0.262 & $<0.001$ & -0.390 & $<0.001$ \\
\hline MSPSS and PCS & -0.164 & 0.312 & 0.009 & 0.918 & 0.129 & 0.184 \\
\hline MSPSS and MCS & 0.507 & 0.001 & 0.241 & 0.003 & 0.332 & $<0.001$ \\
\hline INVR and MSPSS & -0.001 & 0.994 & 0.031 & 0.712 & -0.060 & 0.538 \\
\hline PCS and MCS & 0.150 & 0.354 & 0.062 & 0.454 & -0.034 & 0.724 \\
\hline
\end{tabular}

INVR: Index of Nausea, Vomiting, and Retching; PCS: Physical Component Summary scale; MCS: Mental Component Summary scale; MSPSS:

Multidimensional Scale of Perceived Social Support.

Table 4. Relationships of NVR and perceived social support with physical health

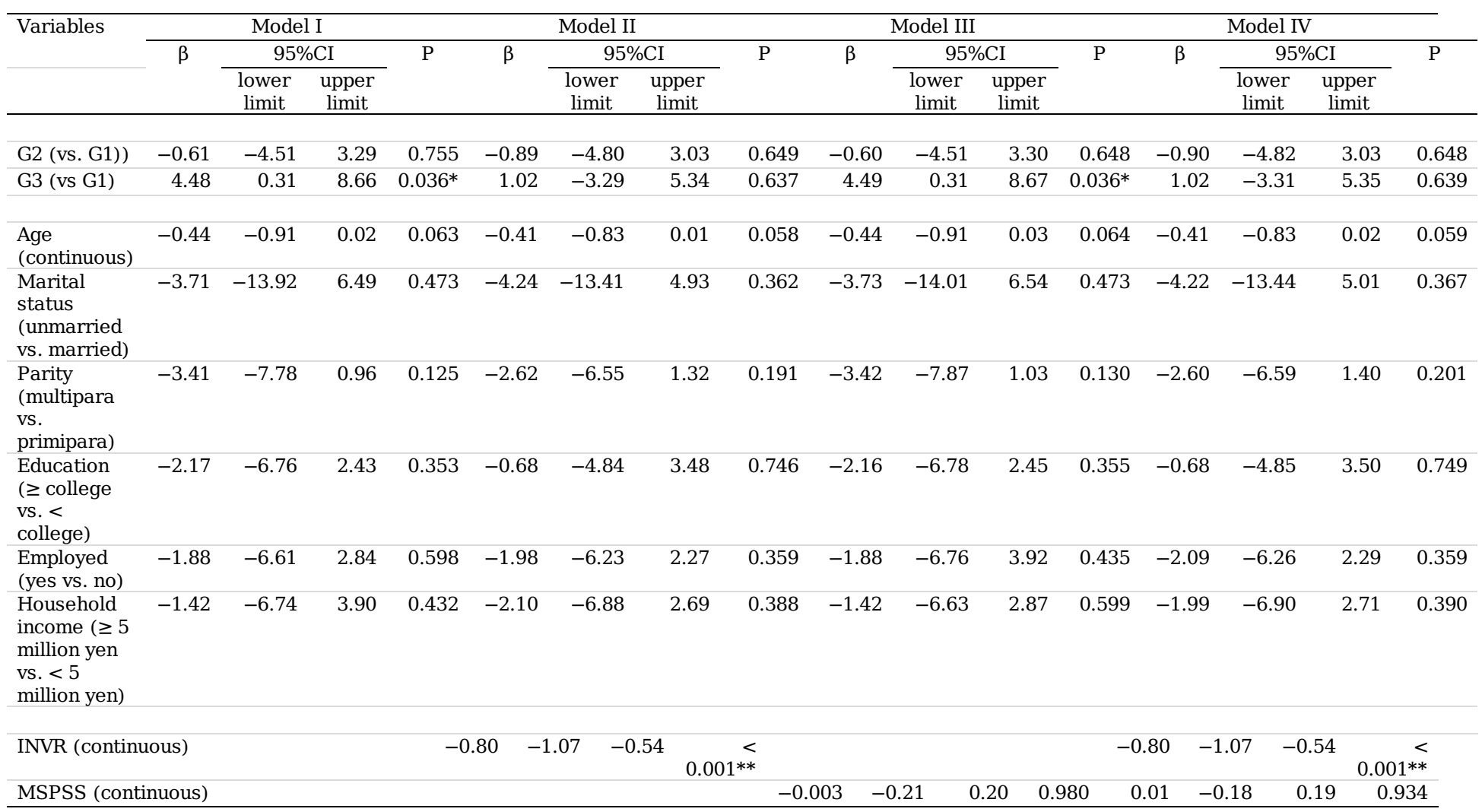

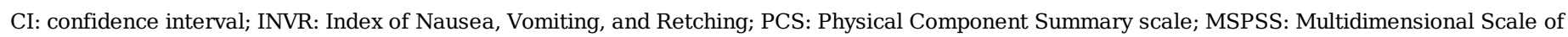
Perceived Social Support.

$* \mathrm{p}<0.05, * * \mathrm{p}<0.001$

Table 5. Relationships of NVR and perceived social support with mental health 


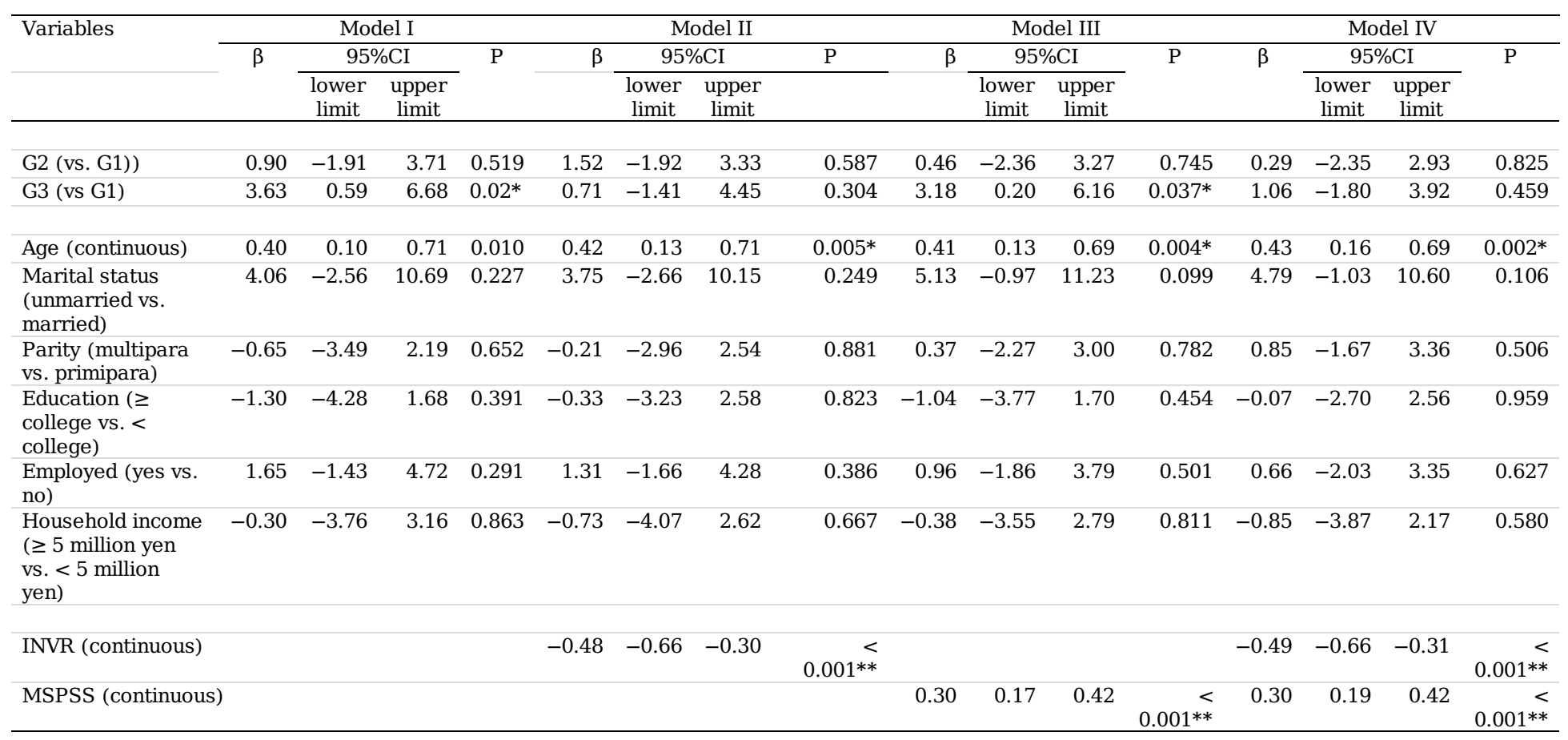

CI: confidence interval; INVR: Index of Nausea, Vomiting, and Retching; PCS: Physical Component Summary scale; MSPSS: Multidimensional Scale of Perceived Social Support.

$* \mathrm{p}<0.05, * * \mathrm{p}<0.001$ 